\title{
Impaired Recollection of Visual Scene Details in Adults with Autism Spectrum
}

\section{Conditions}

Rose A. Cooper ${ }^{1,2}$, Kate C. Plaisted-Grant ${ }^{1}$, Deborah E. Hannula ${ }^{3}$, Charan Ranganath ${ }^{4}$, Simon Baron-Cohen ${ }^{5}$ Jon S. Simons ${ }^{1,2}$

${ }^{1}$ Department of Psychology, University of Cambridge, Cambridge, UK

${ }^{2}$ Behavioural and Clinical Neuroscience Institute, University of Cambridge, UK

${ }^{3}$ Department of Psychology, University of Wisconsin, Milwaukee, Wisconsin, US

${ }^{4}$ Center for Neuroscience and Department of Psychology, University of California, Davis, California, US

${ }^{5}$ Autism Research Centre, Department of Psychiatry, University of Cambridge, Cambridge, UK

\section{Author Note}

Rose A. Cooper, Behavioural and Clinical Neuroscience Institute, Department of Psychology, University of Cambridge, UK; Kate C. Plaisted-Grant, Department of Psychology, University of Cambridge, UK; Deborah E. Hannula, Department of Psychology, University of Wisconsin, Milwaukee, Wisconsin, US; Charan Ranganath, Center for 
Neuroscience, Department of Psychology, University of California, Davis, California, US;

Simon Baron-Cohen, Autism Research Centre, Department of Psychiatry, University of

Cambridge, UK; Jon S. Simons, Behavioural and Clinical Neuroscience Institute, Department of Psychology, University of Cambridge, UK.

This research was supported by a James S. McDonnell Scholar Award to J.S.S., an Economic and Social Research Council Award to R.A.C., and Leverhulme Trust Visiting Professorship and Parke Davis Exchange Fellowship Awards to C.R. We would like to thank all the participants who gave their time for this experiment, as well as the Autism Research Centre, Cambridge for helping with participant recruitment for this study.

Correspondence concerning this article should be addressed to Dr. Jon Simons, Department of Psychology, University of Cambridge, Downing Street, Cambridge CB2 3EB, UK. E-mail: jss30@cam.ac.uk. Phone: +44 1223 333566. Fax: +44 1223764760. 


\begin{abstract}
Subtle memory deficits observed in autism spectrum conditions (ASC) have often been characterised as reflecting impaired recollection and it has been proposed that a relational binding deficit may underlie the recollection impairment. However, subjective recollection and relational binding have not been measured within the same task in ASC to date and it is unclear whether a relational binding deficit can provide a full account of recollection impairments in ASC. Relational memory has also not been compared to item memory when the demands of the two tasks are comparable. To assess recollection, relational memory, and item memory within a single task in ASC, 24 adults with ASC and 24 typically developed adults undertook a change detection memory task that assessed recollection of item-specific and spatial details. Participants studied rendered indoor and outdoor scenes and, in a subsequent recognition memory test, distinguished scenes that had not changed from those that had either undergone an item change (a different item exemplar) or a relational (spatial) change, which was followed by a subjective recollection judgement. The ASC group identified fewer item changes and spatial changes, to a similar degree, which was attributable to a specific reduction in recollection-based recognition relative to the control group. These findings provide evidence that recollection deficits in ASC may not be driven entirely by a relational binding deficit.
\end{abstract}

Key Words: autism; recollection; relational memory; episodic memory 


\section{Impaired Recollection of Visual Scene Details in Adults with Autism Spectrum}

\section{Conditions}

Autism Spectrum Conditions (ASC) are broadly associated with social communication difficulties, unusually repetitive/inflexible behaviours, as well as sensory differences (DSM-5 - American Psychiatric Association, 2013). While not a diagnostic symptom, research has indicated that ASC also tends to be associated with specific differences in performance on long-term memory tasks (see Boucher, Mayes, \& Bigham, 2012; Bowler, Gaigg, \& Lind, 2011, for reviews). Given that long-term memory is central to many aspects of everyday functioning, including social interaction, social cognition and selfidentity, it is important to investigate exactly how and why long-term memory may be different in ASC compared to typically developed individuals.

Two largely overlapping theoretical explanations have been widely used to describe the profile of long-term memory in ASC. The first account highlights an impairment in recollection but a largely intact use of familiarity-based mechanisms (Bigham, Boucher, Mayes, \& Anns, 2010; Boucher, Bigham, Mayes, \& Muskett, 2008), and the other points to a selective impairment in relational binding but preserved item memory (Gaigg, Gardiner, \& Bowler, 2008; Bowler et al., 2011). Recollection is associated with the subjective experience of remembering specific details and contextual information tied to a particular event, whereas familiarity is associated with the feeling of knowing that something has been encountered before, but without recollection of additional, specific details from the original encoding experience (Yonelinas, 2002). In turn, relational binding has been defined as forming relations between constituent aspects of an experience (Konkel \& Cohen, 2009), specifically the relationship between items and their extrinsic context, and is primarily governed by the hippocampus (Diana, Yonelinas, \& Ranganath, 2007; Konkel \& Cohen, 2009), whereas 
memory for specific features of an item, independent of context, can be supported by extrahippocampal regions such as the perirhinal cortex (Awipi \& Davachi, 2008; Davachi, 2006). Binding item and context information is highly important for successful recollection of episodic details, and a deficit in binding relational details has been thought to lead to the subtle recollection impairments observed in ASC.

Evidence for reduced recollection of relational information in ASC has been shown across a number of different tasks. During recall of autobiographical memories, individuals with ASC generally recall fewer specific episodic memory details than matched comparison participants, but they can recall just as many general memory details (Crane \& Goddard, 2008; Crane, Pring, Jukes, \& Goddard, 2012; Lind \& Bowler, 2010; Lind, Williams, Bowler, Peel, \& Raber, 2014; Maister, Simons, \& Plaisted-Grant, 2013). Furthermore, individuals with ASC often have difficulties recalling the 'source' or additional contextual information associated with studied items, but generally show normal item recognition (Bennetto, Pennington, \& Rogers, 1996; Bigham, Boucher, Mayes, \& Anns, 2010; Bowler, Gardiner, \& Berthollier, 2004; Bowler, Gaigg, \& Gardiner, 2014; Hala, Rasmussen, \& Henderson, 2005; Lind \& Bowler, 2009). Such findings are typically interpreted as providing evidence for reduced recollection of relational details, but a typical level of item familiarity. Interestingly, individuals with ASC also tend to benefit more than controls from retrieval cues and 'task support', which lessens demands on recollection (Bowler et al., 2004; Maras \& Bowler, 2012). In addition to reduced objective memory for context details, individuals with ASC have been shown to make fewer self-reported 'remember' (recollection) judgements and more 'know' (familiarity) judgements (Tulving, 1985) when evaluating the basis of their memory for recognised items (Bowler et al., 2000; Bowler, Gardiner, \& Gaigg, 2007; Meyer, 
Gardiner, \& Bowler, 2014), suggesting they are less able to recollect any type of detail associated with the original context in which an item was learnt.

As mentioned, the relational binding account proposes that the aforementioned recollection deficits in ASC can be explained by a hippocampal binding impairment. In studies looking at semantic clustering of words during recall, which has been shown to be related to hippocampal activity (Manning, Sperling, Sharan, Rosenberg, \& Kahana, 2012), individuals with ASC are less likely to cluster words in memory according to semantic relationships, leading to reduced recall of related words but often no difference for unrelated words compared to control participants (Bowler, Gaigg, \& Gardiner, 2008; Gaigg et al., 2008; Maister et al., 2013). Unlike control participants, individuals with ASC do not selectively recall context-relevant aspects of scenes (Loth, Carlos Gómez, \& Happé, 2011), suggesting they are less likely to bind the details encoded to their context. Also consistent with the relational binding theory are findings that individuals with ASC have difficulty with episodic future thinking (Lind \& Bowler, 2010; Lind et al., 2014), fictitious scene construction (Lind et al., 2014), and have reduced memory for object-location associations within a spatial navigation context (Lind, Williams, Raber, Peel, \& Bowler, 2013), all of which require hippocampally-mediated relational binding of elements to form a coherent event (Mullally \& Maguire, 2013). A study that aimed to directly test the relational binding hypothesis assessed memory for item-context conjunctions, and for the item or context elements alone (Bowler et al., 2014) and observed that the ASC group showed typical levels of recognition of single item or context elements but reduced recognition of item-context conjunctions. This evidence suggests that impaired relational binding could provide a good account of memory deficits in ASC. 
Whereas relational binding of some form may be necessary for recollection, due to the need to associate a familiar item with a prior event, it is currently unresolved whether impaired relational binding between an item and its event context fully encompasses the nature of recollection deficits seen in ASC. As discussed above, item memory is largely intact in ASC, and studies of memory (e.g. Gaigg et al., 2008) as well as of perception (e.g. Happé \& Frith, 2006) suggest that individuals with ASC are more likely to perceive and encode item-specific details than contextual details. However, tasks that assess relational memory often require memory for more complex, specific information than tests of item memory, which can be accomplished by a general feeling of familiarity in a standard old-new recognition task. Conversely, when old and new items in a recognition task are visually similar, memory for the item details needs to be much more specific and complex. In this situation, recollection of item-specific details from the original presentation is often necessary to successfully identify an object as new and performance benefits from a 'recall-to-reject' strategy (Kim \& Yassa, 2013; Migo, Montaldi, Norman, Quamme, \& Mayes, 2009). Based on the current evidence in ASC, it is unclear whether item memory deficits might appear when item-specific details need to be recollected and whether recollection deficits extend beyond situations that are heavily reliant on item-context relational encoding.

Other factors might contribute to recollection deficits in ASC, specifically leading to reduced explicit and subjective recollection extending beyond the influence of hippocampal relational binding. For instance, deficits in elaborative encoding and retrieval monitoring, largely governed by the prefrontal cortex (PFC) (Henson, Rugg, Shallice, Josephs, \& Rugg, 1999; Ranganath, Johnson, \& D-Esposito, 2003) might impair the retrieval of episodic details and the experience of recollection. Memory deficits in ASC have been previously related to potential PFC dysfunction (Minshew \& Goldstein, 2001; Minshew \& Williams, 2007), an 
approach that fits nicely with findings of impaired metacognition and monitoring of memory accuracy in ASC (Grainger, Williams, \& Lind, 2014; Wojcik, Moulin, \& Souchay, 2013). Furthermore, memory deficits in ASC have also been related to those following parietal dysfunction (Boucher \& Mayes, 2012), given the posterior parietal cortex (PPC) is known to play a role in the subjective experience of recollection and influences the confidence and vividness with which episodic representations are retrieved (Simons, Peers, Mazuz, Berryhill, \& Olsen, 2010). PPC dysfunction in ASC would potentially explain reduced specificity of episodic memories, reduced 'remember' responses, and a reduced likelihood to retrieve memories from a first-person perspective (Bowler et al., 2007; Lind \& Bowler, 2010). Despite the fact that these regions, connections, and their associated cognitive processes play an important role in the recollection network (Rugg \& Vilberg, 2013), it is currently unclear whether long-term memory differences in ASC could extend beyond a relational binding impairment and more widely affect the ability to subjectively recollect specific details.

To test whether impaired relational binding might be able to fully explain recollection deficits in ASC, and to test item memory under more complex demands, a task developed by Hannula et al. (2010) was adapted. Participants were presented with a series of visual scenes and then, in a later recognition test, judged whether the scene was the same as before, was entirely new, or whether an item in the scene had change in appearance (item memory), or had moved (relational memory). Patients with hippocampal lesions have been reported to exhibit mildly reduced item memory in a version of this task (Hannula et al., 2015), likely because item memory can benefit from an association to the scene context and necessitates associating the new item cue to the original item. Importantly, however, hippocampal patients showed disproportionately larger impairments on the relational memory condition (Hannula et al., 2015), which appeared to place greater demands on relational binding than the item 
memory condition. Thus, this task is useful for assessing a disproportionate context-based relational memory impairment in ASC. In addition, it is important to assess the subjective contribution of recollection to recognition of the scenes, including item and relational changes, to evaluate if a deficit in subjective recollection might affect performance for all types of scene regardless of the varying relational binding demands. This was incorporated into the task by the use of a modified remember-know judgement, by asking participants whether they could consciously remember the specific appearance of the original scene or changed object. Recollection of scenes containing an item change necessitated retrieval of specific item details from the item cue, and recollection of scenes containing a relational change necessitated retrieval of the original context, specifically location, of the item. Correct identification of changed scenes should largely benefit from recollection of the original scene appearance, including item specific and spatial details, whereas the use of recollection to identify scenes as the same should be less frequent (Aly \& Yonelinas, 2012).

Despite the fact that the relational binding and recollection accounts largely overlap, it is possible to derive subtly different predictions within this task based on the literature discussed. A selective relational binding account of memory deficits in ASC would predict a disproportionate impairment on the relational relative to item condition, replicating the pattern, although not necessarily the severity, of performance seen in hippocampal patients. On the other hand, if recollective experience is impaired, over and above relational binding, we would expect equally impaired detection of item and relational changes in the ASC group coupled with a reduction in the use of recollection to support recognition performance across all conditions. As far as we are aware, the current study is the first to assess memory for scenes, including item and relational details, alongside subjective reports of recollection in ASC. 


\section{Method}

\section{Participants}

24 participants with a diagnosis of ASC (11 males) and 24 control participants (11 males) took part in this study. All participants were aged between 18 and 45, and had normal or corrected-to-normal vision and hearing. No participant in the control group had a known current or historical diagnosis of any psychiatric, neurological or developmental condition. Participants in the ASC group all had a formal diagnosis of high-functioning autism $(\mathrm{N}=2)$ or Asperger Syndrome $(\mathrm{N}=22)$ according to DSM-IV or ICD-10 criteria, and received their diagnosis following specialist assessment by a qualified clinician. All participants were administered the Autism Spectrum Quotient (AQ; Baron-Cohen, Wheelwright, Skinner, Martin, \& Clubley, 2001), as well as the Wechsler Abbreviated Scale of Intelligence (WASI; Wechsler, 1999). The AQ is a 50 item questionnaire, measuring self-reported autistic traits in 5 categories: communication, social, imagination, local details, and attention switching, with a maximum score of 50. The WASI provides estimates of verbal and non-verbal IQ via the administration of four sub-tests: Vocabulary, Similarities, Block Design, and Matrix Reasoning. The groups were well matched on age, years of education, verbal IQ (VIQ), nonverbal IQ (PIQ) and full-scale IQ (FSIQ), all $p>$.4. The ASC group scored significantly more highly than the control group on the AQ, $\mathrm{t}(46)=11.1, p<.001$, (see Table 1).

\section{INSERT TABLE 1 ABOUT HERE}

Participants with ASC were recruited via participant databases at the Cambridge Laboratory for Research into Autism and the Autism Research Centre, Cambridge. Control participants were recruited via a participant database held by the Behavioural and Clinical Neuroscience Institute (BCNI), Cambridge University, as well as via social media adverts. Ethical approval for this study was obtained from the Cambridge Psychology Research Ethics 
Committee. Participants gave written informed consent prior to taking part and were paid a standard honorarium for their time.

\section{Materials}

The stimuli used for this experiment were a series of 96 computer-generated indoor and outdoor scenes originally created by Hannula and colleagues (Hannula et al., 2010; Hannula et al., 2015) for their research on item and relational memory. The scenes were 800 x 600 pixels and 3 versions of each scene were used: an original version, a version containing an item change, and a version containing a spatial relational change, leading to 288 scenes in total. Scenes containing item changes were identical to their original versions apart from one item which was replaced by a different but similar exemplar of that item (e.g., the laundry pile changing to an overflowing washing basket, see Figure 1). Scenes containing spatial changes were identical to their originals apart from one item which had been moved to a new, equally plausible location within the scene (e.g., the present moving from the chair to the sofa, see Figure 1). For this latter condition, an equal number of spatial changes were from left to right as from right to left.

\section{Procedure}

Participants completed a computer-based long-term memory change detection task. The task was run using MATLAB (Mathworks Inc.) Cogent 2000 toolbox (http://www.vislab.ucl.ac.uk/cogent_2000.php). Participants completed two experiment phases, a study phase and a test phase, repeated over 4 study-test blocks. Each study phase contained 15 scenes and each test phase contained 20 scenes, including 5 new scenes (NEW) and all 15 studied scenes. Out of the studied scenes one-third remained identical to how they were presented in the study phase (SAME), one-third contained an item change (ITEM), and 
one-third contained a spatial change (SPATIAL). Collapsed across blocks, 60 scenes were encoded and 80 scenes (20 Same, 20 Item, 20 Spatial, and 20 New) were presented during test. To ensure full counterbalancing, 4 test formats were used across participants so that each scene was tested within each condition equally often. The order of the stimuli within each of the 4 blocks was randomised per participant for each study phase and was pseudorandomised in each test phase so that no more than 3 trials in a row were the same type of test scene. The order of the blocks was also randomised for each participant.

During the study phase, each scene was presented for 10 seconds. Participants were instructed to study the scenes with the aim to remember the specific appearance and location of the objects within the scene. Study trials were separated by a blank screen, presented for 500ms (see Figure 1A). Immediately after the study phase and before the test phase, participants were asked to count backwards out loud from a random number displayed on the screen, ranging between 70 and 99 , for 30 seconds, which acted as a short distracter task to discourage rehearsal. For each test trial participants were presented with a scene for 6 seconds and were instructed to use this time first to decide whether they recognised the scene and, if so, whether they thought any object within the scene had changed from the study phase. Four response options then appeared below the scene for a further 4 seconds. These options were "SAME", "ITEM", "SPATIAL", and "NEW", and participants used the number keys 1-4 on the keyboard to respond. The scene was left on the screen throughout the response time to minimise working memory demands and to maximise retrieval support.

If the participant responded "NEW', the program moved straight on to the next trial. "SAME", "ITEM", or "SPATIAL" responses all required participants to make a subjective judgement about whether they could remember the original appearance of the scene or 
changed object (see Figure 1B), by responding "YES" or "NO" to one of the following questions. If the participant responded "SAME", they were asked if they could consciously remember the original scene appearance, including memory for various thoughts or feelings they had about details of the scene appearance or the manner in which they had tried to remember the scene details. If the participant responded "ITEM", they were asked if they could remember what the original item looked like, in terms of specific details of the item that were now different. If the participant responded "SPATIAL", they were asked if they could remember where the item was originally located. Written instructions were given to the participant (see Appendix 1) and the experimenter additionally explained the instructions, providing examples of the types of details that consituted 'remembering'. The instructions were adapted from standard remember-know judgements (e.g. Tulving, 1985), but focused on recollection of the scene appearance, item appearance, or item location. Each trial in the test phase was separated by a blank screen, presented for 500ms.

INSERT FIGURE 1 ABOUT HERE

Participants' understanding of the task instructions was ensured by training on a practice task containing 12 scenes in a study phase and 16 scenes in a test phase, during which they were asked to verbally explain all of their responses to the experimenter both in terms of the condition category they selected and the yes-no responses to the 'remember' questions they made. If the participant made any errors in response justification during the practice task or did not understand part of the instructions, the appropriate part of the task was explained again until it was clear the participant fully understood the instructions. Participants were told that they would be asked to explain how they made their yes-no responses to the 'remember' questions in a questionnaire at the end of the experiment. 
Following completion of the memory task, participants completed a debriefing questionnaire, which verified participants' understanding of the task instructions, the AQ, and the WASI.

\section{Data Analysis}

Data were analysed using SPSS (version 21), and all analyses were conducted using a standard alpha level of .05. All statistics reported are from two-tailed tests. Effect sizes are reported using eta-squared $\left(\eta^{2}\right)$ values for analyses of variance (ANOVAs) and Cohen's $d$ for t-tests. The $95 \%$ confidence interval (CI) of each effect size is also reported. Non-integer degrees of freedom indicate Greenhouse-Geisser correction for non-sphericity. Analyses of Same, Item, and Spatial test scenes were conducted using planned Helmert contrasts, including the Same test scene category as the level 1 contrast, the Item test scene category as the level 2 contrast and the Spatial test scene category as the level 3 contrast.

\section{Results}

\section{Memory for Scenes}

\section{Global Scene Recognition is High in both the ASC and Control Groups}

First, memory for the overall scenes and detection of new scenes was assessed. Oldnew scene discrimination can be achieved by overall scene familiarity and was predicted to be largely intact in ASC. Due to the fact the participants were never asked if they recognised each scene as 'old' but were given the option to identify scenes as New, correct identification of New scenes (i.e., New "hits") and misidentification of studied scenes as 'New' (i.e., New

"false alarms" [FAs]), were combined to produce a corrected measure of performance (hits FAs). Due to negatively skewed data and ceiling effects within both groups, a non-parametric Mann-Whitney $\mathrm{U}$ test was used, revealing a trend towards better old-new scene discrimination in the control group compared to the ASC group, $\mathrm{U}(46)=196, \mathrm{Z}=1.96, p=$ 
.05. Importantly, mean corrected performance was high within both groups (ASC: $0.90, \mathrm{SD}=$ .11 ; control: $0.96, \mathrm{SD}=.06$ ), demonstrating that both groups were able to correctly recognise the overall scenes with very little difficulty.

\section{Memory for Scene Detail}

In the sections that remain, analyses are limited to Same, Item, and Spatial scenes that were correctly endorsed as "old" (i.e. not mistakenly endorsed as "new"), removing any influence of overall scene recognition. Hits (the proportion of correct responses), FAs (the proportion of the other two types of scene that were misattributed to the category in question), and corrected accuracy (hits - FAs) were calculated for Same, Item, and Spatial test scenes. Hits, FAs, and corrected accuracy were also calculated separately for responses based on remembering (a "yes" response to the remember question) and knowing (a "no" response to the remember question).

\section{Memory for both Item and Spatial Scene Detail is Impaired among ASC Participants}

Our first analyses addressed the question of whether ASC performance is disproportionately reduced on the test of spatial relational memory relative to item memory. A 2 (group: ASC, control) x 2 (test scene: Item change, Spatial change) ANOVA on corrected accuracy (see Figure. 2) revealed that the ASC group were worse overall at identifying scene changes than the control group, $\mathrm{F}(1,46)=6.62, p=.01, \eta^{2}=.13, \mathrm{CI}=.01$ .30. There was no effect of condition, $\left(\mathrm{F}<.1, \eta^{2}<.01\right)$, indicating that identification of item and spatial changes were matched for difficulty, and no interaction $\left(\mathrm{F}<.3, \eta^{2}<.01\right)$, providing no evidence of a disproportionate relational binding impairment. The ASC group correctly identified significantly fewer item $(\mathrm{t}(46)=2.58, p=.01, d=0.75, \mathrm{CI}=0.16-1.33)$ and spatial changes $(\mathrm{t}(46)=2.20, p=.03, d=0.64, \mathrm{CI}=0.05-1.21)$, which was due to 
reduced hits (item: $\mathrm{t}(46)=2.80, p<.01, d=0.81, \mathrm{CI}=0.22-1.39$; spatial: $\mathrm{t}(46)=2.14, p=$ $.04, d=0.62, \mathrm{CI}=0.03-1.19)$ and not due to increased FAs for both types of change $($ all $\mathrm{t}<$ 1.1, all $d<0.32$ ). See Table 2 for hits and FAs for each type of test scene.

INSERT TABLE 2 ABOUT HERE

INSERT FIGURE 2 ABOUT HERE

To verify that reduced memory for scene detail was not driven by level of autistic traits or general cognitive functioning in the ASC group, we assessed correlations between AQ and FSIQ scores with Item and Spatial corrected accuracy as well as the difference score between Item and Spatial accuracy (Item-Spatial) in the ASC group. None of the correlations were significant (all $\mathrm{r}<|.24|$, all $p>.27$ ).

\section{Contributions of Recollection to Accurate Scene Differentiation are Reduced in ASC}

Having established that the ASC group were impaired at detection of both Item and Spatial changes, we next investigated the contribution of recollection to performance (remember hits - remember FAs) across all types of test scene. A 2 (group) x 3 (test scene: Same, Item, Spatial) ANOVA on corrected remember responses (see Figure. 3) revealed that recollection differed across the three scene types, $\mathrm{F}(2,92)=17.91, p<.001, \eta^{2}=.28, \mathrm{CI}=$ .13-.41, specifically, recollection-based scene identification was higher for both types of changes than for Same scenes, $\mathrm{F}(2,92)=29.31, p<.001, \eta^{2}=.38, \mathrm{CI}=.17-.55$, and there were more recollection-based identifications of Spatial changes than Item changes, $F(2,92)=$ $6.75, p=.01, \eta^{2}=.13, \mathrm{CI}=.01-.31$. Importantly, the control group made more successful recollection judgements than the ASC group, $\mathrm{F}(1,46)=7.87, p<.01, \eta^{2}=.15, \mathrm{CI}=.01-.33$, 
and this did not differ depending on the type of test scene $\left(\mathrm{F}<.4, \eta^{2}<.01\right)$. Therefore, the ASC group showed a general reduction in subjective recollection-based responses to correctly identify the scenes. Reduced recollection in the ASC group was due to reduced remember hits for Same scenes $(\mathrm{t}(46)=2.86, p<.01, d=0.82, \mathrm{CI}=0.23-1.41)$ and Item scenes $(\mathrm{t}(46)=2.82, p<.01, d=0.82, \mathrm{CI}=0.22-1.40)$, and marginally for Spatial scenes $(\mathrm{t}(46)=1.85, p=.07, d=0.53, \mathrm{CI}=-0.05-1.11)$ compared to the control group. Remember FAs were near floor for Item and Spatial changes, and the proportion of recollection FAs for Same scenes did not differ between the groups $(\mathrm{t}<.3, d<0.07)$.

\section{INSERT FIGURE 3 ABOUT HERE}

To verify that the use of familiarity to identify scenes did not differ between the groups, a 2 (group) x 3 (test scene) ANOVA was conducted on know hits minus know FAs. Corrected know responses differed between the scenes, $\mathrm{F}(2,92)=16.80, p<.001, \eta^{2}=.26$, $\mathrm{CI}=.12-.39:$ Same scenes were more likely to be successfully identified with know responses than changed scenes, $\mathrm{F}(2,92)=17.83, p<.001, \eta^{2}=.27, \mathrm{CI}=.08-.46$, and know responses were more frequent for Item changes than for Spatial changes, $\mathrm{F}(2,92)=14.66, p<$ $.001, \eta^{2}=.24, \mathrm{CI}=.06-.42$. Importantly, there was no difference between the groups $(\mathrm{F}<.1$, $\left.\eta^{2}<.01\right)$ and no interaction between group and test scene $\left(F<1, \eta^{2}<.02\right)$. For Item and Spatial changes, the groups did not differ on either know hits (all $\mathrm{t}<.6$, all $d<.17$ ) or FAs (all $\mathrm{t}<1.5$, all $d<.42$ ), but, when looking at identification of Same scenes, the ASC group made more know hits $(\mathrm{t}(46)=2.87, p<.01, d=0.83, \mathrm{CI}=0.23-1.41)$ and FAs $(\mathrm{t}(46)=3.29$, $p<.01, d=0.95, \mathrm{CI}=0.35-1.54$ ) than the control group (see Table 3 for remember and know hits and FAs for each type of test scene). Therefore, reduced recollection-based detection of changes in the ASC group was accompanied by increased incorrect familiarity-based judgements of changed scenes as the Same. 


\section{INSERT TABLE 3 ABOUT HERE}

\section{Discussion}

The goal of this experiment was to test whether memory deficits in ASC are associated with a disproportionate relational binding impairment or are associated with a broader deficit in subjective recollection. We found that participants in the ASC group were significantly worse at detecting both Item and Spatial changes, to a similar degree, compared to the control group. As shown in the analysis of corrected remember responses, detection of changes, particularly relational changes, largely benefitted from recollection, and the contribution of recollection to recognition was reduced in the ASC group for all types of test scene. Corrected know responses did not differ between the groups, however, the ASC group were more likely to falsely judge changed scenes as the Same based on familiarity. This suggests that even though relational memory was associated with recollection in this task, the ASC group did not show the disproportionate impairment that would be consistent with a hippocampal relational binding deficit. Instead, ASC participants exhibited a general reduction in subjective recollection across all test scenes.

Results from this study add to the body of evidence that indicates reduced recollection of episodic details and relational information in ASC (e.g. Bowler et al., 2014; Crane et al., 2012; Lind et al., 2014; Meyer et al., 2014). However, the results go further by revealing that, beyond memory for the relationship between an item and its context, subjective recollection deficits also affected memory for the scene content and item specific details. Reduced recollection of item appearance is inconsistent with the idea of intact or even superior memory for item-information in ASC, which has important implications for understanding the nature of memory representations in ASC. Previously, it has been assumed that the 
seemingly normal item-specific memory and reduced relational memory in ASC may be influenced by perceptual differences, such as a reduction in perceptual processing of contextual or relational information (Happé \& Frith, 2006), or heightened processing of low level, item-specific perceptual information (Mottron, Dawson, Soulières, Hubert, \& Burack, 2006), and unique properties of objects (Plaisted, O'Riordan, \& Baron-Cohen, 1998).

However, these perceptual differences do not seem to be able to fully explain the pattern of memory differences observed in ASC.

It is possible that our finding of reduced item and relational memory emerged due to the fact that the difficulty and complexity of the item and relational change conditions were matched. This is in contrast to previous studies that measured item and relational memory in ASC. For instance, specific vs. general autobiographical-episodic details have been used to measure relational and item memory respectively (Maister et al., 2013), and item memory has also been measured in terms of memory for only one element whereas relational memory has been measured as memory for multiple elements (e.g. Bowler et al., 2014). In both of these examples, relational memory is necessarily more detailed than item memory. Additionally, differences in test procedure, such as item recognition and relational or source recall (Bowler et al., 2004; Massand \& Bowler, 2013) provide greater retrieval support for item memory than relational memory, with the former relying less on recollection than the latter (Yonelinas, 2002). Results from the current study are consistent with the possibility that the aforementioned reports of impaired recollection of relational, contextual information in ASC could have been driven by factors other than impaired relational binding, affecting the experience of recollection and specificity with which information can be encoded and retrieved. 
It is important to acknowledge that that the item and relational conditions used in the current task are by no means process pure. Given that the items are embedded within scenes, it is likely that memory for item details would have benefitted from binding the item with the scene context (for example, by relating a particular feature of an item to another object in the scene (Hannula et al., 2010)). In fact, the presence of scene context has been shown to benefit detection of both item and spatial changes (Hollingworth, 2007), and activity in the hippocampus is associated with detection of item and spatial changes in scenes (Duncan, Ketz, Inati, \& Davachi, 2012), perhaps reflecting the quantity of information recollected Therefore, it would be interesting to test whether the presence of scene context affected recollection of scene details in the same way for ASC and control participants to further examine whether impaired relational binding may account for our findings. Additionally, to fully test a relational binding account, it would also be important to assess relational familiarity and implicit memory in ASC, perhaps via a forced-choice test format, even though the idea that relationally bound representations can be retrieved without recollection is debateable (see Mayes, Montaldi, \& Migo, 2007 for a discussion of this issue). Evidence of brain structural differences within the hippocampus and its relationship to memory in ASC is mixed (see Goh \& Peterson, 2012 for a review), but lends some support to the idea that disruption of hippocampal relational binding might play a role in reduced recollection in ASC.

The present results are unlikely to be fully explained, however, in terms of hippocampal dysfunction. A previous study demonstrated that patients with hippocampal lesions have impaired detection of item and relational spatial changes, but have a disproportionate deficit in detection of spatial changes (Hannula et al., 2015). This disproportionate deficit was interpreted to reflect a role for the hippocampus in relational 
binding, because identification of spatial changes is likely to be more dependent on relational binding than detection of item changes (even if item-context binding is important for both). In contrast to patients with hippocampal damage, our ASC group showed no evidence of a disproportionate deficit in detection of spatial changes. These findings suggest that the recollection deficit in ASC reflects, at least in part, extrahippocampal dysfunction. A recent study by Gaigg, Bowler, Ecker, Calvo-Merino, \& Murphy (2015), which used fMRI to investigate relational binding and subjective recollection in ASC, is consistent with this assertion. Although the authors concluded that impaired relational binding may explain reduced subjective recollection in ASC, their findings showed that a consistent reduction in 'remember' responses in ASC was not moderated by the relational binding demands of the task, as well as no evidence for attenuated hippocampal activity during relational encoding and no evidence that attenuated hippocampal activity led to reduced remember responses. Instead, activity of the inferior frontal gyrus was not selectively enhanced for remember relative to know responses in the ASC group unlike in the control group, suggesting a possible role for PFC dysfunction in reduced recollection in ASC.

The aforementioned finding is consistent with observations of atypical PFC activity during encoding of later recognised objects in ASC (Greimel et al., 2012). Furthermore, the hippocampus has been shown to be sensitive to relational memory regardless of whether subjective recollection also occurs, whereas the PFC and hippocampal-PFC connectivity play an important role in explicit recollection (Hannula \& Ranganath, 2009). PFC dysfunction and reduced connectivity to posterior regions has been suggested to explain the pattern of memory deficits observed in ASC (Minshew \& Goldstein, 2001; Minshew \& Williams, 2007), affecting encoding strategies implemented as well as the amount and specificity of information that can be maintained during encoding and retrieval. This is consistent with 
evidence of reduced PFC activity and working memory deficits in ASC, especially when task demands are complex (e.g. Vogan et al., 2014; see Barendse et al., 2013 for a review). Additionally, individuals with ASC are less able to initiate strategic encoding strategies, which has been shown to lead to reduced recollection-based 'remember' responses compared to controls (Meyer et al., 2014), and it is possible that subtle impairments in spontaneous expressive language seen in ASC (Boucher, 2012) might contribute to impairments in elaborative encoding. Importantly, PFC dysfunction might explain the marginally reduced scene recognition in the ASC group. Impaired recognition has been occasionally reported in ASC (Bowler et al., 2004; Massand \& Bowler, 2013), and familiarity impairments are also more apparent in low-functioning individuals (Boucher et al., 2008). Whereas the ability to organize, monitor and maintain specific information may most greatly affect recollection, PFC dysfunction in ASC may also impact on familiarity-based recognition when the stimuli and task demands are complex. A more severe pattern of impairment has been observed in schizophrenia patients tested on a version of the current task (Hannula et al., 2010), suggesting that PFC dysfunction may affect memory similarly across other disorders. However, unlike the ASC group, schizophrenia patients showed increased false recognition of scene changes, emphasising that differences across disorders is an interesting area for future studies to consider.

PFC dysfunction could also lead to poor retrieval monitoring, as indicated by impaired metamemory in ASC (Grainger et al., 2014) and impaired retrieval cue specification, as indicated by an increased reliance on retrieval support (Bowler et al., 2004). This is in line with the proposal that deficits in self-projection, involving abilities such as prospection and theory of mind and driven by medial prefrontal regions (Buckner \& Carroll, 2007), may contribute to reduced recollection in ASC (Lind et al., 2014). To test whether 
difficulty with retrieval monitoring and self-projection might contribute to recollection of scene details, future studies could use a cueing procedure in which the item that may have been changed is highlighted during the test (e.g., Luck \& Vogel, 1997), limiting the search process and increasing task support. A difficulty with retrieval is also in accordance with the idea that memory deficits in ASC might relate to dysfunction within parietal regions, specifically the PPC (Boucher \& Mayes, 2012), due to its involvement in the retrieval of specific, vivid details from memory, contributing to the experience of subjective recollection (Simons et al., 2010), known to be reduced in ASC (e.g. Lind et al., 2014). While there have been no direct studies assessing functional and structural differences within parietal areas in ASC, the PPC, along with medial temporal regions and the PFC, is known to be part of the default mode network, playing an important role in episodic memory retrieval (Sestieri, Corbetta, Romani, \& Shulman, 2011). Evidence has pointed to both reduced connectivity and atypical activity within the default mode network in ASC, particularly frontal-parietal interactions (e.g. Just, Keller, Malave, Kana, \& Varma, 2012). Therefore, information monitoring, subjective recollection, and their neural bases are important areas to further research in relation to long-term memory deficits in ASC.

In addition to those already discussed, one potential limitation of the current task is that the ASC group were less likely to attend to the to-be-changed object during the study phase, leading to reduced recognition of changes. However, studies using complex scenes or video clips, employing changes akin to those used in the current study, have reported little difference in perceptual change detection between individuals with ASC and controls (AuYeung, Benson, Castelhano, \& Rayner, 2011; Fletcher-Watson, Leekam, Turner, \& Moxon, 2006; Loth, Carlos Gómez, \& Happé, 2008), or have shown enhanced change detection in ASC (Fletcher-Watson et al., 2012; Smith \& Milne, 2009), meaning it is unlikely that the 
ASC participants would not have attended to the changed object at all within our encoding time frame. Importantly, failing to attend to the to-be-changed object at all could not readily explain the reduced use of recollection to identify scenes, especially those that were the Same where recollection did not depend on memory for a single object within the scene.

Nonetheless, the precise pattern of attention across the scenes at encoding may have differed between the ASC and control groups, contributing to a general recollection impairment. Eye movements during encoding of complex stimuli, such as objects and scenes, have been shown to influence subsequent recollection-based memory, where recollection of has been associated with more fixations and more clustered fixations at encoding than subsequent familiarity (Kafkas \& Montaldi, 2011; Sharot, Davidson, Carson, \& Phelps, 2008). Given that individuals with ASC have been shown to exhibit different patterns of attention in response to natural stimuli such as scenes, including attending to different parts of the scene (e.g. Loth et al., 2011), and reduced duration of fixations (e.g. Anderson, Colombo, \& Shaddy, 2006), the influence of attention on recollection is an important area for future research in ASC to consider. Furthermore, due to the use of a subjective remember judgement rather than eliciting narrative responses, we were unable to assess the content of recollected experiences. Variations in attention and encoding strategies might lead to subtle differences in recollected content between individuals with ASC and typical individuals, such as the type of information, quantity, and precision, which is important for future studies to consider. It is also important to use research into the nature of memory impairments, such as the current study, and potential causes of memory impairments to inform the development of teaching strategies, that should provide support for learning and retrieving information in a detailed, elaborative, and flexible way. 


\section{Conclusions}

This study aimed to assess subjective recollection of visual scene details, including item-context relations and item-specific details, in ASC to test whether recollection deficits are driven by a disproportionate relational binding impairment. Individuals with ASC found item and relational changes equally difficult to detect, which was accompanied by a general reduction in subjective recollection. While relational binding is highly important for recollection, the results from the present study suggest that memory deficits in ASC may also be driven by other factors, affecting the specificity with which memories are encoded and retrieved and the experience of recollection more generally. Future studies should further explore how attention and different encoding and retrieval strategies affect memory in ASC. This would be facilitated by investigating the neural mechanisms underpinning encoding and retrieval processes in ASC and under what circumstances they can be moderated, which will help us to understand why specific memory deficits occur in this population.

\section{References}

Aly, M., \& Yonelinas, A. P. (2012). Bridging consciousness and cognition in memory and perception: evidence for both state and strength processes. PloS One, 7(1), e30231. American Psychiatric Association. (2013). Diagnostic and statistical manual of mental disorders: DSM-5. Washington, D.C: American Psychiatric Association.

Anderson, C. J., Colombo, J., \& Shaddy, D. J. (2006). Visual scanning and pupillary responses in young children with autism spectrum disorder. Journal of Clinical and Experimental Neuropsychology, 28(7), 1238-56.

Au-Yeung, S. K., Benson, V., Castelhano, M., \& Rayner, K. (2011). Eye Movement Sequences during Simple versus Complex Information Processing of Scenes in Autism Spectrum Disorder. Autism Research and Treatment, 657383. 
Awipi, T., \& Davachi, L. (2008). Content-specific source encoding in the human medial temporal lobe. Journal of Experiment Psychology: Learning, Memory, and Cognition, 34, 769-79.

Barendse, E. M., Hendriks, M. P. H., Jansen, J. F. A, Backes, W. H., Hofman, P. A. M., Thoonen, G., Kessels, R. P. C., \& Aldenkamp, A. P. (2013). Working memory deficits in high-functioning adolescents with autism spectrum disorders: neuropsychological and neuroimaging correlates. Journal of Neurodevelopmental Disorders, 5(1), 14.

Baron-Cohen, S., Wheelwright, S., Skinner, R., Martin, J., \& Clubley, E. (2001). The autismspectrum quotient (AQ): evidence from Asperger syndrome/high-functioning autism, males and females, scientists and mathematicians. Journal of Autism and Developmental Disorders, 31(1), 5-17.

Bennetto, L., Pennington, B., \& Rogers, S. (1996). Intact and impaired memory functions in autism. Child Development, 67(4), 1816-35.

Bigham, S., Boucher, J., Mayes, A., \& Anns, S. (2010). Assessing recollection and familiarity in autistic spectrum disorders: methods and findings. Journal of Autism and Developmental Disorders, 40(7), 878-89.

Boucher, J., Bigham, S., Mayes, A., \& Muskett, T. (2008). Recognition and language in low functioning autism. Journal of Autism and Developmental Disorders, 38(7), 1259-69.

Boucher, J., \& Mayes, A. (2012). Memory in ASD: have we been barking up the wrong tree? Autism, 16(6), 603-11.

Boucher, J., Mayes, A., \& Bigham, S. (2012). Memory in autistic spectrum disorder. Psychological Bulletin, 138(3), 458-96.

Bowler, D. M., Gaigg, S. B., \& Gardiner, J. M. (2008). Effects of related and unrelated context on recall and recognition by adults with high-functioning autism spectrum disorder. Neuropsychologia, 46(4), 993-9. 
Bowler, D. M., Gaigg, S. B., \& Gardiner, J. M. (2014). Binding of Multiple Features in Memory by High-Functioning Adults with Autism Spectrum Disorder. Journal of Autism and Developmental Disorders, 44(9), 2355-62.

Bowler, D. M., Gaigg, S. B., \& Lind, S. E. (2011). Memory in Autism : binding, self and brain. In Researching the autism spectrum: contemporary perspectives (pp. 316-346). Cambridge: Cambridge University Press.

Bowler, D. M., Gardiner, J. M., \& Berthollier, N. (2004). Source Memory in Adolescents and Adults with Asperger's Syndrome. Journal of Autism and Developmental Disorders, $34(5), 533-42$.

Bowler, D. M., Gardiner, J. M., \& Gaigg, S. B. (2007). Factors affecting conscious awareness in the recollective experience of adults with Asperger's syndrome. Consciousness and Cognition, 16(1), 124-43.

Bowler, D. M., Gardiner, J. M., \& Grice, S. J. (2000). Episodic memory and remembering in adults with Asperger syndrome. Journal of Autism and Developmental Disorders, 30(4), $295-304$.

Buckner, R. L., \& Carroll, D. C. (2007). Self-projection and the brain. Trends in Cognitive Sciences, 11(2), 49-57.

Crane, L., \& Goddard, L. (2008). Episodic and semantic autobiographical memory in adults with autism spectrum disorders. Journal of Autism and Developmental Disorders, 38(3), $498-506$.

Crane, L., Pring, L., Jukes, K., \& Goddard, L. (2012). Patterns of autobiographical memory in adults with autism spectrum disorder. Journal of Autism and Developmental Disorders, 42(10), 2100-12.

Davachi, L. (2006). Item, context and relational episodic encoding in humans. Current Opinion in Neurobiology, 16, 693-700. 
Diana, R. A., Yonelinas, A. P., \& Ranganath, C. (2007). Imaging recollection and familiarity in the medial temporal lobe: a three-component model. Trends in Cognitive Sciences, 11(9), 379-86.

Duncan, K., Ketz, N., Inati, S., \& Davachi, L. (2012). Evidence for area CA1 as a match/mismatch detector: A high resolution fMRI study of the human hippocampus. Hippocampus, 22(3), 389-98.

Fletcher-Watson, S., Leekam, S. R., Connolly, B., Collis, J. M., Findlay, J. M., McConachie, H., \& Rodgers, J. (2012). Attenuation of change blindness in children with autism spectrum disorders. The British Journal of Developmental Psychology, 30(Pt 3), 446-58.

Fletcher-Watson, S., Leekam, S. R., Turner, M. a, \& Moxon, L. (2006). Do people with autistic spectrum disorder show normal selection for attention? Evidence from change blindness. British Journal of Psychology, 97(Pt 4), 537-54.

Gaigg, S. B., Gardiner, J. M., \& Bowler, D. M. (2008). Free recall in autism spectrum disorder: the role of relational and item-specific encoding. Neuropsychologia, 46(4), 983-92.

Gaigg, S. B., Bowler, D. M., Ecker, C., Calvo-Merino, B., Murphy, D. G. (2015). Episodic Recollection Difficulties in ASD Result from Atypical Relational Encoding: Behavioral and Neural Evidence. Autism Research, doi: 10.1002/aur.1448.

Goh, S., \& Peterson, B. S. (2012). Imaging evidence for disturbances in multiple learning and memory systems in persons with autism spectrum disorders. Developmental Medicine \& Child Neurology, 54(3), 208-13.

Grainger, C., Williams, D. M., \& Lind, S. E. (2014). Metacognition in highfunctioning adults with autism spectrum disorder: Diminished feelings of knowing and mindreading ability. Journal of Abnormal Psychology, 123(3), 650-9. 
Greimel, E., Nehrkorn, B., Fink, G. R., Kukolja, J., Kohls, G., Müller, K., ... Schulte-Rüther, M. (2012). Neural mechanisms of encoding social and non-social context information in autism spectrum disorder. Neuropsychologia, 50(14), 3440-9.

Hala, S., Rasmussen, C., \& Henderson, A. M. E. (2005). Three types of source monitoring by children with and without autism: the role of executive function. Journal of Autism and Developmental Disorders, 35(1), 75-89.

Hannula, D. E., \& Ranganath, C. (2009). The Eyes Have It: Hippocampal Activity Predicts Expression of Memory in Eye Movements. Neuron, 63, 1-8.

Hannula, D. E., Ranganath, C., Ramsay, I. S., Solomon, M., Yoon, J., Niendam, T. A., ... Ragland, J. D. (2010). Use of eye movement monitoring to examine item and relational memory in schizophrenia. Biological Psychiatry, 68(7), 610-6.

Hannula, D. E., Tranel, D., Allen, J. S., Kirchhoff, B. A., Nickel, A. E., \& Cohen, N. J. (2015). Memory for Items and Relationships among Items Embedded in Realistic Scenes: Disproportionate Relational Memory Impairments in Amnesia. Neuropsychology, 29(1), 126-38.

Happé, F., \& Frith, U. (2006). The weak coherence account: detail-focused cognitive style in autism spectrum disorders. Journal of Autism and Developmental Disorders, 36(1), 525 .

Henson, R. N. A, Rugg, M. D., Shallice, T., Josephs, O., Dolan, R. J. (1999). Recollection and Familiarity in Recognition Memory: An Event-Related Functional Magnetic Resonance Imaging Study. The Journal of Neuroscience, 19(10), 3962-72.

Hollingworth, A. (2007). Object-Position Binding in Visual Memory for Natural Scenes and Object Arrays. Journal of Experimental Psychology: Human Perception and Performance, 33(1), 31-47. 
Just, M. A., Keller, T. A., Malave, V. L., Kana, R. K., \& Varma, S. (2012). Autism as a neural systems disorder: A theory of frontal-posterior underconnectivity. Neuroscience \& Biobehavioural Reviews, 36(4), 1292-313.

Kafkas, A., \& Montaldi, D. (2011). Recognition memory strength is predicted by pupillary responses at encoding while fixation patterns distinguish recollection from familiarity. Quarterly Journal of Experimental Psychology, 64(10), 1971-89.

Kim, J., \& Yassa, M. A. (2013). Assessing recollection and familiarity of similar lures in a behavioral pattern separation task. Hippocampus, 23(4), 287-94.

Konkel, A., \& Cohen, N. J. (2009). Relational memory and the hippocampus: representations and methods. Frontiers in Neuroscience, 3(2), 166-74.

Lind, S. E., \& Bowler, D. M. (2009). Recognition memory, self-other source memory, and theory-of-mind in children with autism spectrum disorder. Journal of Autism and Developmental Disorders, 39(9), 1231-9.

Lind, S. E., \& Bowler, D. M. (2010). Episodic memory and episodic future thinking in adults with autism. Journal of Abnormal Psychology, 119(4), 896-905.

Lind, S. E., Williams, D. M., Bowler, D. M., Peel, A., \& Raber, J. (2014). Episodic memory and episodic future thinking impairments in high-functioning autism spectrum disorder: An underlying difficulty with scene construction or self-projection? Neuropsychology, $28(1), 55-67$.

Lind, S., Williams, D., Raber, J., Peel, A., \& Bowler, D. M. (2013). Spatial navigation impairments among intellectually high-functioning adults with autism spectrum disorder: Exploring relations with theory of mind, episodic memory, and episodic future thinking. Journal of Abnormal Psychology, 122(4), 1189-99.

Loth, E., Carlos Gómez, J., \& Happé, F. (2008). Detecting changes in naturalistic scenes: contextual inconsistency does not influence spontaneous attention in high-functioning 
people with autism spectrum disorder. Autism Research: Official Journal of the International Society for Autism Research, 1(3), 179-88.

Loth, E., Carlos Gómez, J., \& Happé, F. (2011). Do High-Functioning People with Autism Spectrum Disorder Spontaneously Use Event Knowledge to Selectively Attend to and Remember Context-Relevant Aspects in Scenes? Journal of Autism and Developmental Disorders, 41, 945-61.

Luck, S., \& Vogel, E. (1997). The capacity of visual working memory for features and conjunctions. Nature, 193, 279-81.

Maister, L., Simons, J. S., \& Plaisted-Grant, K. (2013). Executive functions are employed to process episodic and relational memories in children with autism spectrum disorders. Neuropsychology, 27(6), 615-27.

Manning, J. R., Sperling, M. R., Sharan, A., Rosenberg, E. A., \& Kahana, M. J. (2012). Spontaneously reactivated patterns in frontal and temporal lobe predict semantic clustering during memory search. Journal of Neuroscience, 32(26), 8871-8.

Maras, K. L., \& Bowler, D. M. (2012). Context reinstatement effects on eyewitness memory in autism spectrum disorder. British Journal of Psychology, 103(3), 330-42.

Massand, E., \& Bowler, D. M. (2013). Atypical Neurophysiology Underlying Episodic and Semantic Memory in Adults with Autism Spectrum Disorder. Journal of Autism and Developmental Disorders, 45(2), 298-315.

Mayes, A., Montaldi, D., \& Migo, E. (2007). Associative memory and the medial temporal lobes. Trends in Cognitive Sciences, 11(3), 126-35.

Meyer, B. J., Gardiner, J. M., \& Bowler, D. M. (2014). Directed Forgetting in HighFunctioning Adults with Autism Spectrum Disorders. Journal of Autism and Developmental Disorders, 44(10), 2514-24. 
Migo, E., Montaldi, D., Norman, K. a, Quamme, J., \& Mayes, A. (2009). The contribution of familiarity to recognition memory is a function of test format when using similar foils. Quarterly Journal of Experimental Psychology (2006), 62(6), 1198-215.

Minshew, N. J., \& Goldstein, G. (2001). The Pattern of Intact and Impaired Memory Functions in Autism. Journal of Child Psychology and Psychiatry, 42(8), 1095-101.

Minshew, N. J., \& Williams, D. L. (2007). The New Neurobiology of Autism. Neurological Review, 64(7), 945-50.

Mottron, L., Dawson, M., Soulières, I., Hubert, B., \& Burack, J. (2006). Enhanced perceptual functioning in autism: an update, and eight principles of autistic perception. Journal of Autism and Developmental Disorders, 36(1), 27-43.

Mullally, S. L., \& Maguire, E. A. (2013). Memory, Imagination, and Predicting the Future: A Common Brain Mechanism? The Neuroscientist : A Review Journal Bringing Neurobiology, Neurology and Psychiatry, 20(3), 220-34.

Plaisted, K., O’Riordan, M., \& Baron-Cohen, S. (1998). Enhanced discrimination of novel, highly similar stimuli by adults with autism during a perceptual learning task. Journal of Child Psychology and Psychiatry, 39(5), 765-75.

Ranganath, C., Johnson, M. K., D’Esposito, M. (2003). Prefrontal activity associated with working memory and episodic long-term memory. Neuropsychologia, 41, 378-89.

Rugg, M. D., \& Vilberg, K. L. (2013). Brain networks underlying episodic memory retrieval. Current Opinion in Neurobiology, 23(2), 255-60.

Sestieri, C., Corbetta, M., Romani, G. L., \& Shulman, G. L. (2011). Epsiodic memory retrieval, parietal cortex, and the Default Mode Network: functional and topographic analyses. Journal of Neuroscience, 31(12), 4407-20.

Sharot, T., Davidson, M. L., Carson, M. M., \& Phelps, E. A. (2008). Eye movements predict recollective experience. PloS One, 3(8), e2884. 
Simons, J. S., Peers, P. V., Mazuz, Y. S., Berryhill, M. E., \& Olsen, I. R. (2010). Dissociation between memory accuracy and memory confidence following bilateral parietal lesions. Cerebral Cortex, 20, 479-85.

Smith, H., \& Milne, E. (2009). Reduced change blindness suggests enhanced attention to detail in individuals with autism. Journal of Child Psychology and Psychiatry, and Allied Disciplines, 50(3), 300-6.

Tulving, E. (1985). Memory and Consciousness. Canadian Psychology, 26(1), 1-12.

Vogan, V. M., Morgan, B. R., Lee, W., Powell, T. L., Smith, M. L., \& Taylor, M. J. (2014). The neural correlates of visuo-spatial working memory in children with autism spectrum disorder: effects of cognitive load. Journal of Neurodevelopmental Disorders, 6(1), 19.

Wechsler, D. (1999). Wechsler Abbreviated Scale of Intelligence. New York, NY: Psychological Corporation.

Wojcik, D. Z., Moulin, C. J. A., \& Souchay, C. (2013). Metamemory in Children With Autism: Exploring "Feeling-of-Knowing" in Episodic and Semantic Memory. Neuropsychology, 27(1), 19-27.

Yonelinas, A. P. (2002). The Nature of Recollection and Familiarity: A Review of 30 Years of Research. Journal of Memory and Language, 46(3), 441-517. 


\section{Appendices}

Appendix 1: Instructions for the 'remember' questions conditional upon SAME, ITEM, or SPATIAL responses.

"If you recognise a scene (and, therefore, decided to respond 1, 2, or 3) you will then be asked if you can remember what the original version of the scene shown in the study phase looked like.

If you responded 'SAME': You will be asked "Can you remember what the original scene looked like?"

You should respond 'YES' if you can consciously remember how the scene looked when it was presented in the study phase and believe that everything has remained the same, whereas you should respond 'NO' if you cannot remember exactly what the scene originally looked like but nevertheless believe that the scene is the same.

If you responded 'ITEM': You will be asked "Can you remember what the original item looked like?"

You should respond 'YES' if you can consciously remember how the item that you believe has changed looked in the study phase, including memory for specific features, whereas you should respond 'NO' if you believe a particular item has changed in appearance but can't remember how the item originally looked in the study phase.

If you responded 'SPATIAL': You will be asked "Can you remember where the item was originally located?"

You should respond 'YES' if you can consciously remember seeing the item in a different location originally, including memory for its specific relative location, whereas you should respond 'NO' if you believe a particular item has moved but can't remember seeing it in a different location originally." 
Figure 1

\section{A) Study Phase}
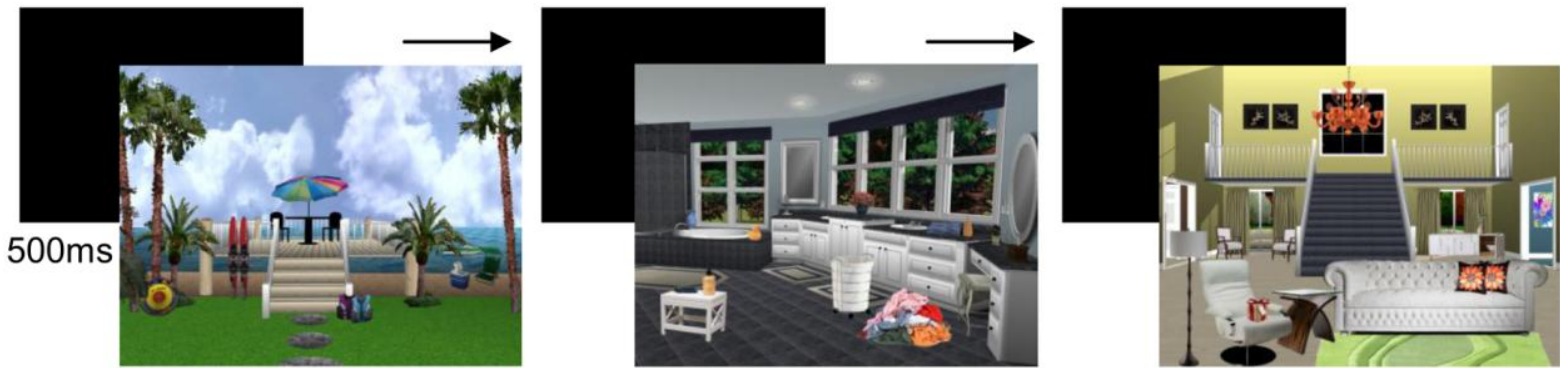

10 seconds

\section{B) Test Phase}

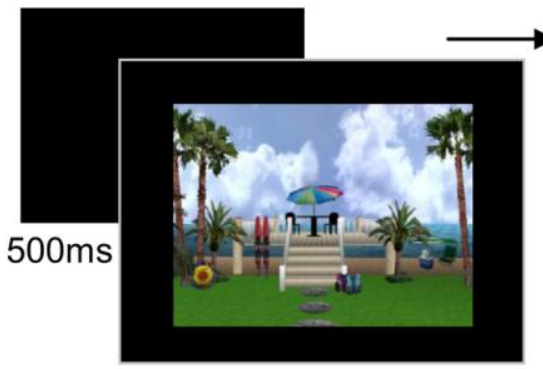

6 seconds

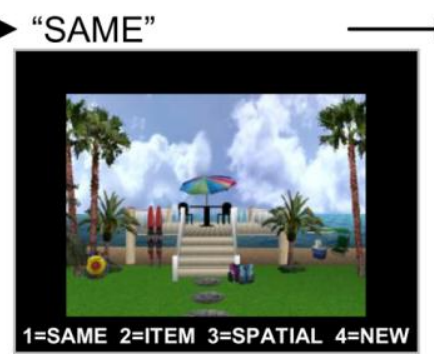

until response (max 4 seconds)

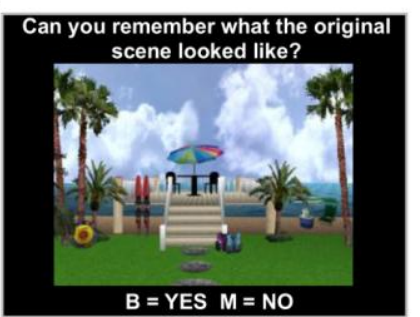

until response (max 4 seconds)

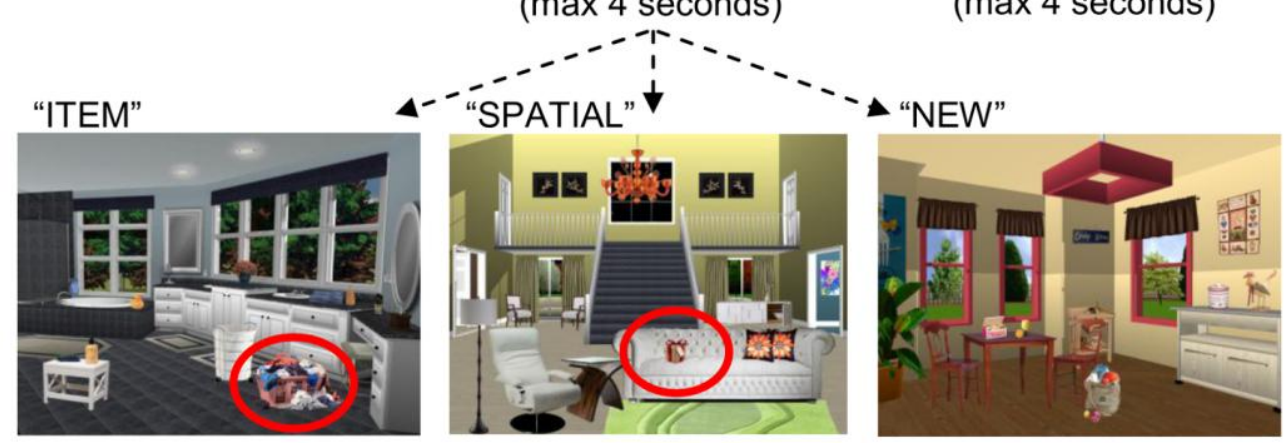

Figure 1. During the study phase (A), participants were shown a series of scenes, each for 10 seconds, separated by a blank screen for 500ms. For each trial in the test phase (B), participants were presented with the scene for 6 seconds, after which the response options appeared and they had an additional 4 seconds in which to make their response. The scene could either be the Same as the studied scene (trial shown), or could contain an Item or Spatial change (changes are circled in the figure for illustration purposes), or the scene could be New. Following identification of the scene as the Same or changed (Item or Spatial), participants were asked to indicate whether their response was based on recollection or not 
Figure 2

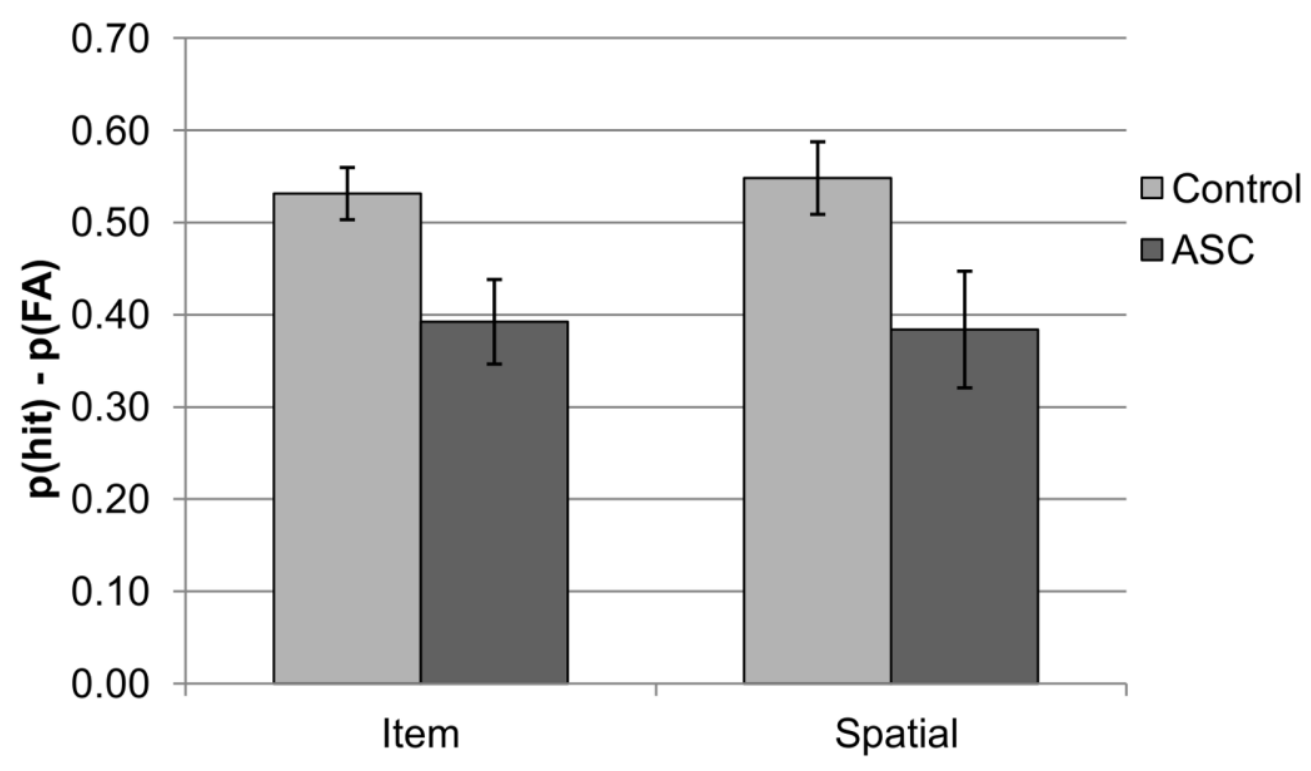

Figure 2. Corrected accuracy (hits - FAs) for Hits for Same, Item change, and Spatial change scenes. The ASC showed significantly reduced identification of both Item and Spatial changes compared to the control group, showing no evidence for a disproportionate impairment in identifying Spatial changes. Error bars = SEM 
Figure 3

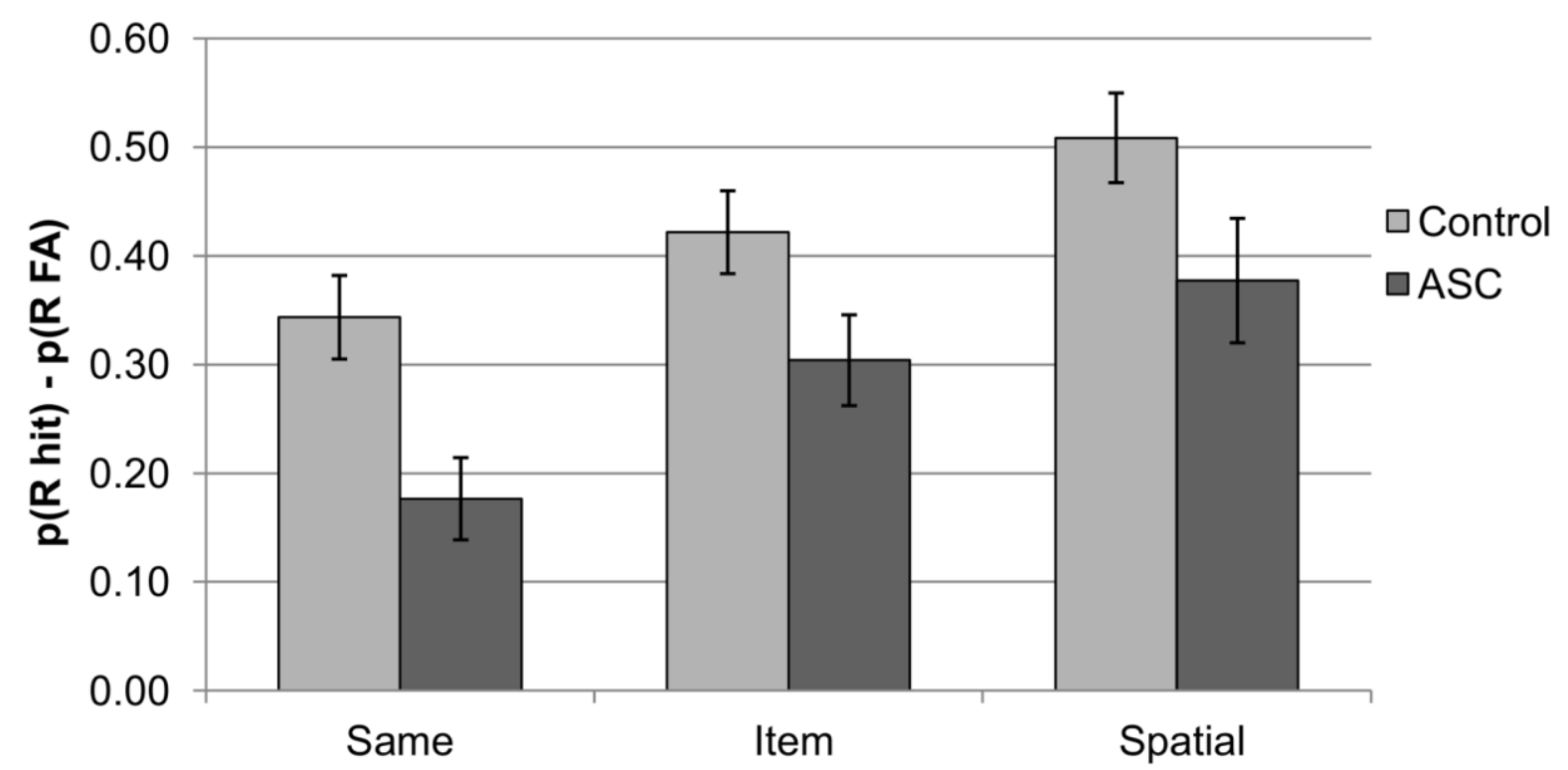

Figure 3. Corrected remember responses (R Hits - R FAs) for Same, Item change, and Spatial change scenes. Recollection was used to identify more changed scenes than Same scenes, and also more Spatial changes than Item changes. The ASC group made fewer remember-based judgements to identify all types of test scene compared to the control group. Error bars $=$ SEM 
Table 1: Mean (SD) demographic information and psychometric test scores within each group

$$
\operatorname{ASC}(\mathrm{N}=24) \quad \text { Control }(\mathrm{N}=24)
$$

\begin{tabular}{lcc}
\hline Age & $31.75(7.58)$ & $31.00(6.51)$ \\
Years of Education & $15.75(2.35)$ & $16.25(1.94)$ \\
AQ & $37.17(6.40)$ & $17.46(5.88)$ \\
VIQ & $112.00(9.14)$ & $111.71(7.93)$ \\
PIQ & $116.88(8.45)$ & $117.63(8.71)$ \\
FSIQ & $116.33(8.63)$ & $116.54(7.61)$ \\
\hline
\end{tabular}

Note. SD = Standard Deviation; AQ = Autism Quotient; VIQ = Verbal IQ; PIQ = Performance IQ; FSIQ = Full scale IQ 
Table 2: Mean (SD) Hits and FAs for Same, Item change, and Spatial change scenes within each group
ASC
Control

\begin{tabular}{l|lll|lll}
\hline & Same & Item & Spatial & Same & Item & Spatial \\
Hits & $.72(.15)$ & $.56(.22)$ & $.48(.26)$ & $.73(.11)$ & $.71(.13)$ & $.62(.19)$ \\
FAs & $.36(.19)$ & $.17(.11)$ & $.09(.09)$ & $.24(.11)$ & $.17(.08)$ & $.07(.06)$ \\
\hline
\end{tabular}


Table 3: Mean (SD) 'remember' and 'know' Hits and FAs for Same, Item change, and Spatial change scenes within each group
ASC
Control

\begin{tabular}{l|lll|lll}
\hline & Same & Item & Spatial & Same & Item & Spatial \\
R Hits & $.28(.22)$ & $.32(.21)$ & $.39(.28)$ & $.45(.20)$ & $.49(.19)$ & $.52(.20)$ \\
R FAs & $.10(.11)$ & $.02(.04)$ & $.02(.03)$ & $.10(.08)$ & $.06(.07)$ & $.02(.02)$ \\
K Hits & $.44(.21)$ & $.24(.17)$ & $.08(.08)$ & $.28(.19)$ & $.22(.14)$ & $.09(.08)$ \\
K FAs & $.26(.18)$ & $.15(.11)$ & $.07(.08)$ & $.13(.07)$ & $.11(.07)$ & $.05(.05)$ \\
\hline
\end{tabular}

Note. $\mathrm{R}=$ remember (recollection); $\mathrm{K}$ = know (familiarity) 